\title{
WAVEFORM GENERATION IN POWER ELECTRONIC CONVERTER USING OBJECT ORIENTED PROGRAMMING IN MATLAB
}

\section{Dr. jai p agrawal, Purdue University Northwest}

Jai P. Agrawal is a professor in electrical and computer engineering technology at Purdue University Northwest. He received his Ph.D. in electrical engineering from University of Illinois, Chicago, in 1991, dissertation in power electronics. He also received M.S. and B.S. degrees in electrical engineering from Indian Institute of Technology, Kanpur, India, in 1970 and 1968, respectively. His expertise includes analog and digital electronics design, power electronics, nanophotonics, and optical/wireless networking systems. He has designed several models of high frequency oscilloscopes and other electronic test and measuring instruments as an entrepreneur. He has delivered invited short courses in Penang, Malaysia and Singapore. He is also the author of a textbook in power electronics, published by Prentice-Hall, Inc. His professional career is equally divided in academia and industry. He has authored several research papers in IEEE journals and conferences. His current research is focused on renewable energy technology, smart energy grid Phasor analytical tools in ac circuit analysis. 


\title{
WAVEFORM GENERATION IN POWER ELECTRONIC CONVERTER USING OBJECT ORIENTED PROGRAMMING IN MATLAB
}

\begin{abstract}
The paper presents the programming methodology for generating voltage and current waveforms in power electronic converters using MATLAB. Although these waveforms can also be obtained by using MULTISIM or PLEXIM simulation tools, but using MATLAB increases understanding of the circuit operation in steady state better. In this method, the power electronic converter circuit is first resolved into circuit modes (states) based on the specifications and switching strategy employed. The inductor currents and capacitor voltages are used as objects with properties and methods in each circuit state. The terminal values of each circuit state are matched with beginning values of the objects in the next circuit state. Details of classes, methods and functions will be presented in the full paper. The paper presents examples of Buck, Boost, BuckBoost, and other converter topologies. This methodology is general enough to generate waveforms in several DC-DC converters and inverters.
\end{abstract}

\section{INTRODUCTION}

The steady state operation of power converter is fairly complex and require clear understanding of circuit configuration which may not necessarily be constant over a period of time. Analysis and Spice simulation are two well-known methods of obtaining the time-domain waveforms of voltages and currents in different parts of the circuit. The later method is fairly accurate but does not provide enough insight in the circuit operation. Time-domain analysis method serves that purpose. Since the power converters contain a network of switches which may assume multiple configurations giving rise to piece-wise configurable circuits and transitions among them. It is extremely important to identify the circuit configurations, the sequence and the modus operandi of transitions from one configurations to another. The overall voltage and current waveforms on all components including the switches are extremely useful in obtaining the average, peak and ratings and stresses. In this paper, we present a methodical analysis of the circuit configurations, present a generalized method which can use MATLAB based method of generating waveforms of the steady state operation of converters. We employ state-variable methods for analyzing the circuit operation.

A word about its usefulness in education. This method may help in understanding the converter operation. It may be specially helpful when there is soft switching, zero voltage or zero current situations. Other areas where it can be useful when the closed form solution does not exist. This method can be used with initial estimated solution and then converge to solutions in few iterations. This methodology is more useful for senior students who have taken an introductory class in power electronics and control systems and signals.

\section{POWER CONVERTER OPERATION}

This paper concerns the steady-state analysis of Single Source Single Output (SISO) power converters. The power converter is a network of switches, resistor R, energy storage elements such as the inductor L, capacitor C and transformers. Switches are comprised of diodes and transistors. The source may be a dc or ac voltage or current source. The sink is a dc or ac voltage or current sink. The voltage sink is a large capacitor holding constant voltage during the steady state of operation of the converter. The current sink is a large inductor capable of holding constant current during the steady state operation of the converter. The converter operation consist of transient and steady state parts. In this paper, we will concern with the steady state operation of a power converter. 


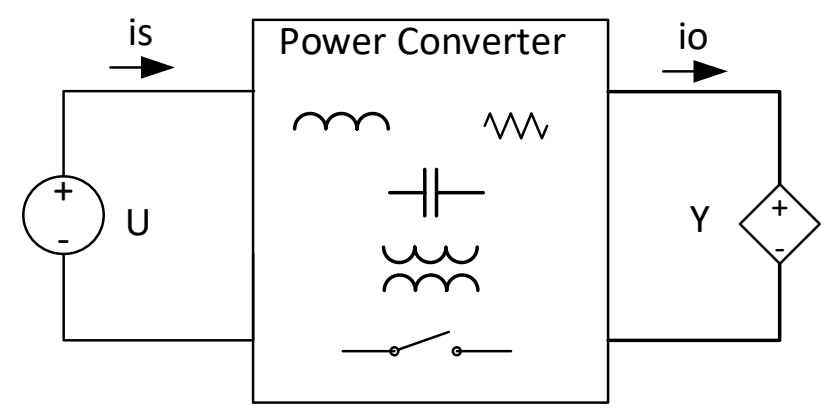

Fig. 1 SISO Power Converter

Transistors and diodes are used as unidirectional electronic switches. Transistors are turned on and off periodically by external time-driven switches and also by the reversal of current direction. The diodes are turned on when voltage across the diode terminals are forward biased, and turned off when the current flowing through them reverses or the voltage across them reverses the polarity, that is, the diodes are activated based on the currents or voltages in the circuit operation. The inductor current $\mathrm{i}_{\mathrm{L}}$ and the capacitor voltage $\mathrm{v}_{\mathrm{C}}$ are the two variables which usually serve as the control of diode or diode network.

If there is only one time-based switch $\mathrm{S}$, the converter operation comprises of two circuit modes: one when the switch is turned on and the other when the switch is turned off. If there are two time-based switches $S_{1}$ and $S_{2}$, the converter operation may comprise of four circuit modes. If one switch $\mathrm{S} 1$ is time based and the other $\mathrm{S}_{2}$ is based on the circuit conditions such as the current inversion or the polarity inversion of a voltage, the converter operation may consist of two, three or four circuit modes.

Each circuit mode is comprised of a network of R, L and C and an excitation $\mathrm{U}$ which is comprised of the sources, sinks and initial conditions on L and C's.

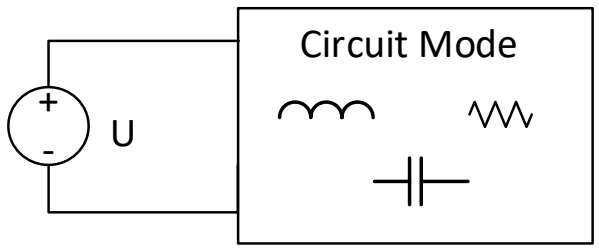

\section{Fig. 2 A generic circuit mode topology in the steady state operation of the SISO power converter}

During the operation of circuit mode, the state variables to be watched are the inductor currents and capacitor voltages. Knowledge thereof will enable us to obtain current and voltage waveforms on all other components of the converter. A circuit mode starts at $t_{0}$ and ends at $t_{1}$ or $t_{2}$, when one of the two conditions is met first, as depicted in Fig. 3:

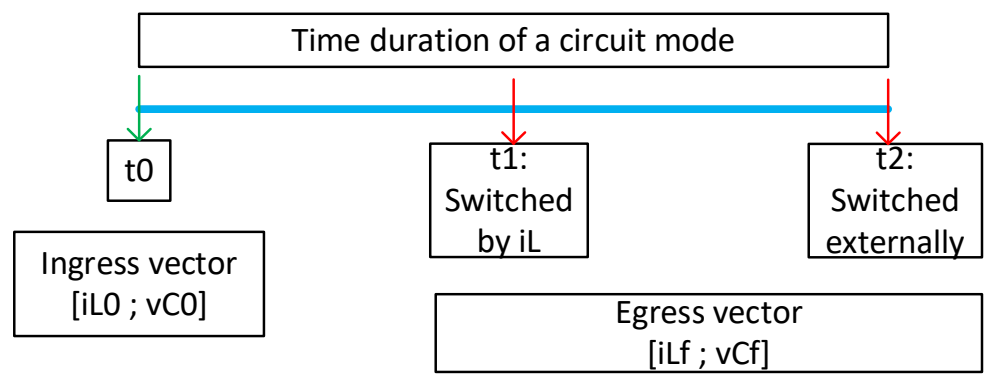

Fig. 3 Operation of a circuit mode in the power converter 
The Ingress vector contains the initial state of the inductor currents and capacitor voltages at the instant $\mathrm{t}_{0}$. The Egress vector indicates their final states. The ending time $t_{1}$ or $t_{2}$ becomes the beginning time in the next mode in sequence of the converter operation. The Egress vector of circuit mode 1 becomes the Ingress vector of the next sequential circuit mode 2 and so on. The Egress vector of last circuit mode in the converter operation must match the Ingress vector of the circuit mode 1.

\section{A CIRCUIT MODE IN THE OPERATION OF THE POWER CONVERTER}

\subsection{A Typical Circuit Mode}

The power converter topology is a network consisting of sources, sinks, R, L, C and switches. Depending upon the state of switches, the converter assumes several circuit modes. A typical circuit mode in a dc-dc converter may look like the following:

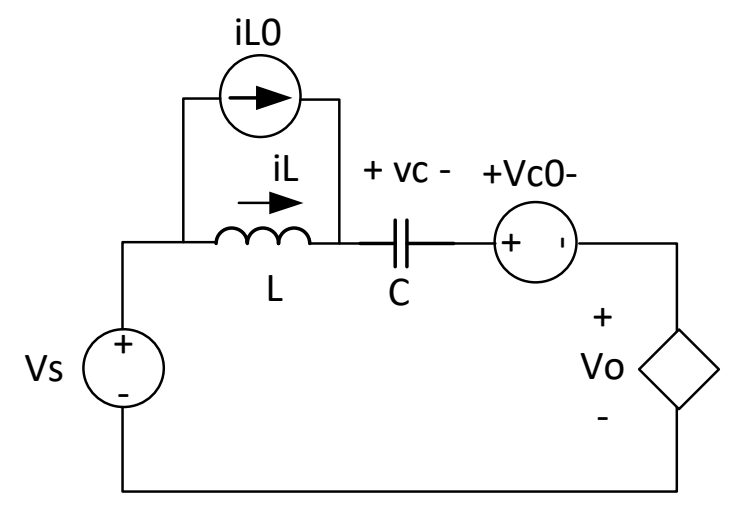

Fig. 4 A typical Circuit Mode configuration

A circuit mode has a time duration from $t_{0} t_{t} t_{f}$. At any instant $t$ in the time interval $t_{0}-t_{f}$, the inductor current $i_{L}$ and the capacitor voltage $\mathrm{v}_{\mathrm{C}}$ are the two state variables of interest. We will enunciate them as two objects under the class state. Let us look at the state variable representation of the circuit at any instant of time t, given by,

$$
\left[\begin{array}{c}
\frac{d i_{L}}{d t} \\
\frac{d v_{C}}{d t}
\end{array}\right]=\left[\begin{array}{cc}
0 & -\frac{1}{L} \\
\frac{1}{C} & 0
\end{array}\right]\left[\begin{array}{l}
i_{L} \\
v_{C}
\end{array}\right]+\left[\begin{array}{c}
\frac{1}{L}\left(V_{S}-V_{O}-V_{C 0}\right) \\
\frac{1}{C} i_{L 0}
\end{array}\right]
$$

In the generic form, $\quad \dot{X}=A X+B$

Where $\mathrm{X}$ is a vector of objects under the state class, $\dot{\mathrm{X}}$ is the vector of derivatives of state objects, matrix A describes the circuit network and $\mathrm{B}$ is the excitation vector involving the sources and the initial conditions of the state variables.

The solution of state variables is obtained in the Laplace domain from,

$$
X(s)=(s I-A)^{-1} B+\mathrm{x} 0
$$

$\mathrm{x} 0$ is vector of the initial values of state variables. Time domain solution is obtained from the impulse response of $\mathrm{X}(\mathrm{s})$,

$$
\left[\begin{array}{l}
I_{L} \\
V_{C}
\end{array}\right]=\left[\begin{array}{cc}
s & \frac{1}{L} \\
-\frac{1}{C} & s
\end{array}\right]^{-1}\left[\begin{array}{c}
\frac{1}{s L}\left(V_{S}-V_{O}-V_{C 0}\right) \\
\frac{1}{s C} i_{L 0}
\end{array}\right]+\left[\begin{array}{c}
\frac{1}{s} i_{L 0} \\
\frac{1}{s} V_{C 0}
\end{array}\right]
$$




$$
\left[\begin{array}{l}
i_{L} \\
v_{C}
\end{array}\right]=\text { invlaplace }\left\{\left[\begin{array}{cc}
s & \frac{1}{L} \\
-\frac{1}{C} & s
\end{array}\right]^{-1}\left[\begin{array}{c}
\frac{1}{s L}\left(V_{S}-V_{O}-V_{C 0}\right) \\
\frac{1}{s C} i_{L 0}
\end{array}\right]\right\}+\left[\begin{array}{c}
i_{L 0} \\
v_{C 0}
\end{array}\right]
$$

We can define state vector $\mathrm{X}$ consisting of arrays of magnitudes at an array of times during the interval $t_{1}$ to $t_{2}$ as the solution of the circuit mode:

$\mathbf{X}=$ state(mag, time) $\quad$ where $\quad \mathrm{X} . \mathrm{mag}=[$ value 1 , value $2, \ldots$ value $\mathrm{n}]$ and $\mathrm{X} . \mathrm{time}=[\mathrm{t} 0, \mathrm{t} 0+\mathrm{dt}, \ldots . \mathrm{t} 2]$.

The initial state of vector object $\mathrm{X}$ in a circuit mode is called the Ingress state Ing:

$$
\text { Ing }=\left[\begin{array}{c}
i_{L 0} \\
v_{C 0}
\end{array}\right]=\left[\begin{array}{l}
\text { state }\left(\left|i_{L 0}\right|, t_{0}\right) \\
\text { state }\left(\left|V_{C 0}\right|, t_{0}\right)
\end{array}\right]
$$

The final state of vector object $\mathrm{X}$ in the circuit mode is called the Egress state Eg:

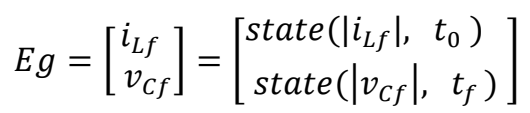

The following MATLAB code is written for a dc-dc converter (Buck, Boost,....) circuit mode consisting of a single state variable, inductor current $\mathrm{i}_{\mathrm{L}}$ :

\subsection{State Class}

The converter topology is a network consisting of sources, sinks, R, L, C and switches. The inductor current $\mathrm{i}_{\mathrm{L}}$ and the capacitor voltage $\mathrm{v}_{\mathrm{C}}$ are the two variables of interest in a converter topology. We will use them as the objects under a class state. The state class have two properties: magnitude and the time at which the state has that value.

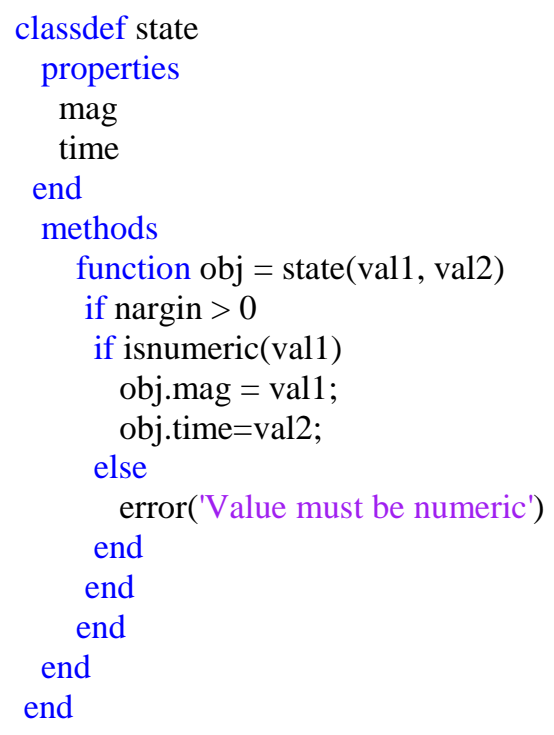

For example, an inductor current, when enunciated as an object under the class state, having a value of $10 \mathrm{~mA}$ at time of 2 milliseconds, is coded as,

$$
\text { iL=state(10e-3, 2e-3). }
$$

An object can also have an unknown value, indicated by NaN, not a number.

An object of class state can also have arrays of properties. For example, the iL object can have an array of 5 values at an array of 5 times, 
$\mathrm{iL}=$ state([-1, -3, 3, -4, 2], [0, 1, 2, 3, 4]).

The state objects can be organized as an array of objects, or a matrix of objects.

\subsection{Time-Based Switching of a Circuit Mode}

The MATLAB function for invoking the circuit mode calculation of state variable vector in mode 1 :

$$
\begin{array}{r}
\text { X1 } 1=\text { cmode_1b(Ing1, A, B1, Eg1, dt); } \\
\text { where Ing }=\left[\begin{array}{c}
i_{L 0} \\
v_{C 0}
\end{array}\right]=\left[\begin{array}{c}
\operatorname{state}\left(\left|i_{L 0}\right|, t_{0}\right) \\
\operatorname{state}\left(\left|V_{C 0}\right|, t_{0}\right)
\end{array}\right] \\
E g=\left[\begin{array}{c}
i_{L f} \\
v_{C f}
\end{array}\right]=\left[\begin{array}{cc}
\operatorname{state}(\text { nan, } & \left.t_{f}\right) \\
\operatorname{state}(\text { nan, } & \left.t_{f}\right)
\end{array}\right]
\end{array}
$$

The 'nan' refers to 'not a number', signifying it to be an unknown value. The $t_{0}$ and $t_{f}$ are respectively the externally controlled beginning and the ending times.

\subsection{Zero Current Switching of a Circuit Mode}

The MATLAB function for invoking the circuit mode calculation of state variable vector in mode 1:

$$
\begin{aligned}
& \text { X1=cmode_1b(Ing1, A, B1, Eg1, dt); } \\
& \text { where Ing }=\left[\begin{array}{c}
i_{L 0} \\
v_{C 0}
\end{array}\right]=\left[\begin{array}{c}
\operatorname{state}\left(\left|i_{L 0}\right|, t_{0}\right) \\
\operatorname{state}\left(\left|V_{C 0}\right|, t_{0}\right)
\end{array}\right] \\
& E g=\left[\begin{array}{l}
i_{L f} \\
v_{C f}
\end{array}\right]=\left[\begin{array}{ll}
\operatorname{state}(0, & \left.t_{f}\right) \\
\operatorname{state}(\text { nan, } & \left.t_{f}\right)
\end{array}\right]
\end{aligned}
$$

The ' 0 ' in the magnitude of final state of the current signifies that the the mode operation ends when the inductor becomes zero for the first time. The capacitor voltage magnitude is specified as 'nan', the unknown value. The ending time $t_{f}$ indicates that the circuit mode will continue up to time $t_{f}$ if the zero current switching does not occur.

\subsection{MATLAB Code for A Circuit Mode}

function S = cktmode1( Ing, A, B, Eg, dt)

\%Ing and Eg are respectively the initial and final state vectors in the circuit mode

$\% \mathrm{~A}$ is the state matrix for the circuit mode

$\% \mathrm{~B}$ is the excitation vector for the circuit mode that includes all sources and initial conditions on the inductor and

\%capacitors.

$\% \mathrm{dt}$ is the incremental step time in the operation of the circuit mode.

$[\mathrm{N}, \mathrm{N}]=\operatorname{size}(\mathrm{A})$;

$\mathrm{I}=$ eye $(\mathrm{N})$;

$\mathrm{s}=\mathrm{tf}(\mathrm{s})$;

$\mathrm{Xs}=\operatorname{inv}(\mathrm{s} * \mathrm{I}-\mathrm{A}) * \mathrm{~B} / \mathrm{s}$;

$\mathrm{t}=$ Ing.time:dt:Eg.time;

S.mag=(Ing.mag+impulse(Xs,(t-t(1))))';

S.time=t;

\%to find the first time instant at which the inductor current becomes zero or less

if isnan(Eg.mag)

if (Ing.mag $<0$ )

$\mathrm{k}=$ find(S.mag $<=$ Eg.mag);

\%Laplace variable s

$\%$ The time array in a circuit mode \%size of the A matrix for the network of the circuit mode

\%Identity matrix for the network of the circuit mode

\%The solution of the state vector $\mathrm{X}$ during the circuit mode

\%The array of magnitude of state vector S in the circuit mode

\%The array of time of state vector $\mathrm{S}$ in the circuit mode

\%Check if the magnitude in the final state vector Eg is not a 'nan'

\%Check if the magnitude in the initial state vector Ing is less than 0

\%k is the array of the instants at which the vector $\mathrm{S}$ is less than or equal to Eg.mag 


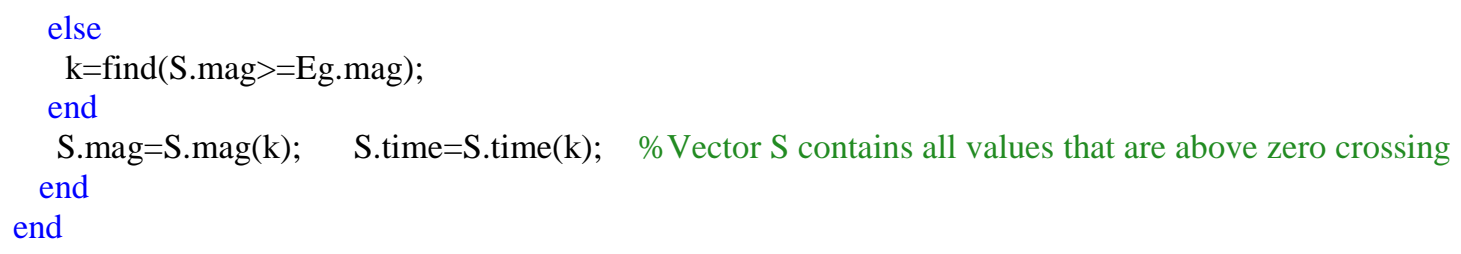

\section{EXAMPLES OF DC-DC CONVERTER}

In this section, we shall take up the examples of steady state operation of Buck dc to dc converter in both Continuous and Discontinuous Conduction modes and a Flyback dc to dc converter in the continuous Conduction modes. Both these converter topologies are single energy storage element, therefore, simpl;e to analyze and obtain the waveforms. The procedure is, however, generic and applies to all converters with one or more energy storage elements.

\subsection{Buck Converter}

The following example shows a 20V to 12V DC-DC Buck Converter operating at $200 \mathrm{kHz}$ and an inductor of 24 $\mu \mathrm{H}$. Find the duty ratio $\mathrm{D}$ at which the converter must operate to deliver $20 \mathrm{~W}$ power to the resistive load at a voltage of $12 \mathrm{~V}$.

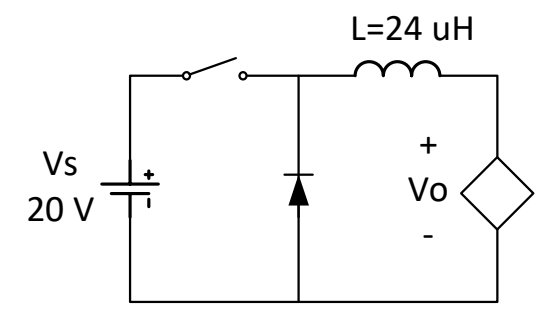

Fig. 5 Buck Converter Topology

Figure 5 shows the topology of the Buck converter. The switch is turned on and off periodically at a switching frequency $f_{S}$. The on period of the switch is $D . T_{S}$ where $D$ is the duty ratio of the on-time to the switching period $T_{S}$. The output voltage is modeled by a voltage sink $V_{O}$ which remains (required to be) constant in steady state for all load currents (in or out). Depending on the delivered output power, the converter operates in two modes: one in which the inductor current is never zero and the other in which it becomes zero for some duration in a period. The first is called the Continuous Conduction Mode (CCM) and the other the Discontinuous Conduction Mode (DCM). The first mode occurs at higher power $\mathrm{P}_{\mathrm{O}}$ and the other occurs at lower power. This paper assumes that the design equations are known which are listed as below:

$$
\text { Output Current: } \quad I_{O}=\frac{P_{O}}{V_{O}} \quad \text { Load Resistance: } \quad R_{L}=\frac{V_{O}^{2}}{P_{O}}
$$

To determine if the converter would operate in CCM or in DCM, we should calculate the load resistance at the boundary between the CCM and the $\mathrm{DCM}^{[1]}{ }^{[2]}$ :

$$
R_{L B}=\frac{2 L f_{S}}{\left(1-\frac{V_{O}}{V_{S}}\right)}
$$

Next, we determine the duty ratio and the initial inductor current in the circuit mode $1^{[1]}{ }^{[2]}$.

The converter operates in CCM if $R_{L} \leq R_{B}$

Duty ratio for the desired output voltage is:

$$
\begin{aligned}
& D=\frac{V_{O}}{V_{S}} \\
& I_{r p p}=\frac{V_{S} D(1-D)}{L f_{S}} \\
& I_{L 0}=I_{O}-\frac{I_{r p p}}{2}
\end{aligned}
$$$$
\text { Peak to peak ripple current in the inductor }{ }^{[1][2]} \text { : }
$$

Initial value of the inductor current in the circuit mode 1: $\quad I_{L 0}=I_{O}-\frac{I_{r p p}}{2}$ 
The converter operates in DCM if $R_{L}>R_{B}$

$$
\begin{array}{ll}
\text { Duty ratio for the desired output voltage }{ }^{[2]}: & D=\sqrt{\frac{2 P_{O} L f_{S}}{V_{S}\left(V_{S}-V_{O}\right)}} \\
\text { Initial value of the inductor current in the circuit mode 1: } & I_{L 0}=0
\end{array}
$$

The MATLAB code consists of two parts. The Part 1 of the code uses various formulae derived from theories presented

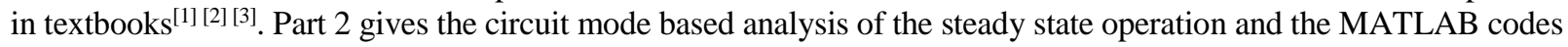
for determining the voltages and current waveforms. Readers are expected to have the Part 1 knowledge before going over to Part 2.

\section{MATLAB Code}

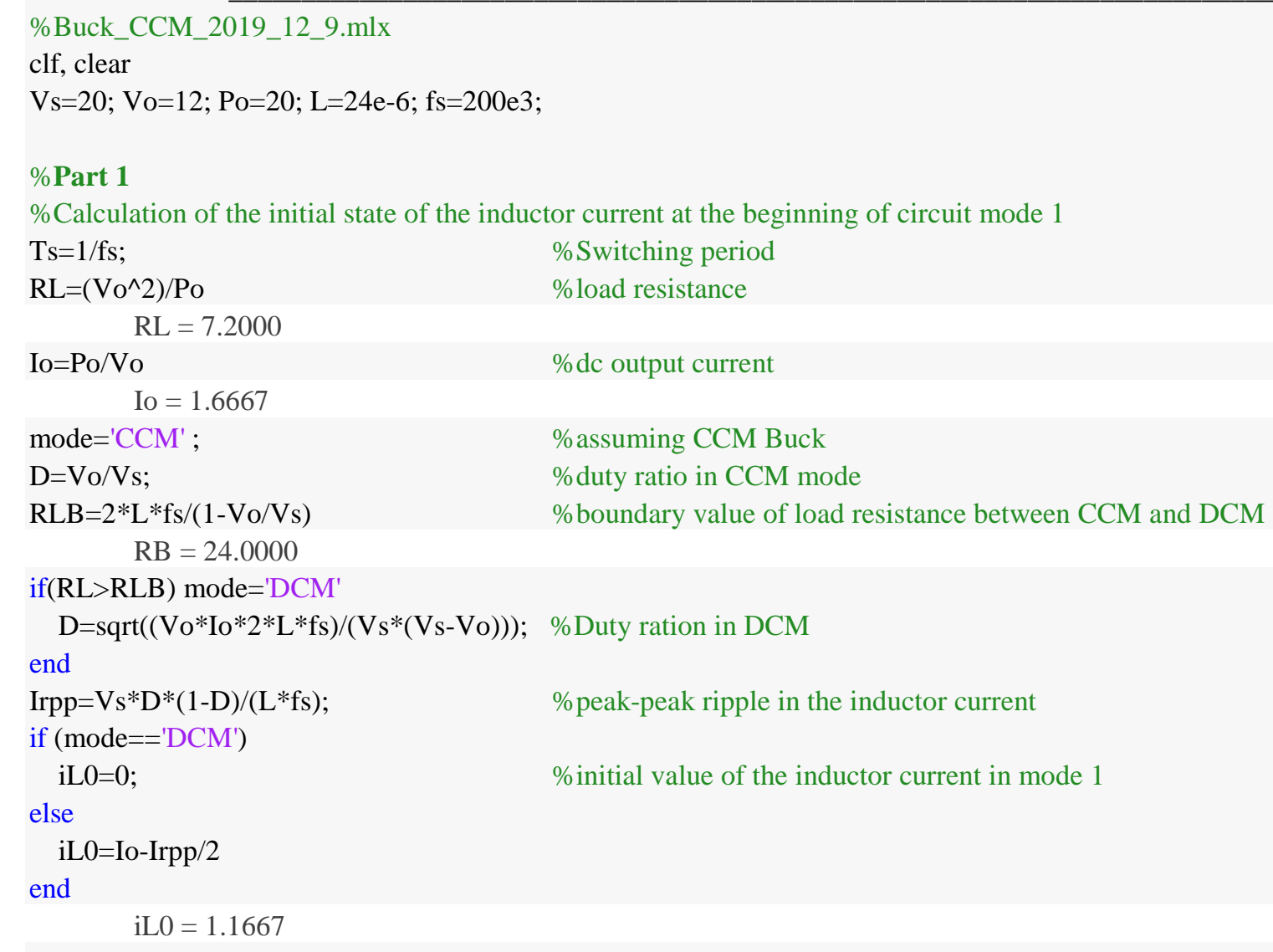

The converter operation in CCM is discussed in the next section.

\subsubsection{Buck in Continuous Conduction Mode (CCM) Operation (Output Power $\mathrm{P}_{\mathrm{O}}=20$ Watts)}

The Buck converter in CCM operates in two configurations: circuit mode 1 when the switch is turned on and remains on. The second circuit configuration occurs when the switch is turned off for the duration of (1-D)Ts. The circuit configuration and the current flow in circuit mode 1 is shown by the thick dark line in the first circuit in Fig. 6 . The circuit configuration and the current flow in circuit mode 2 is shown by the thick dark line in the second circuit in Fig. 6. Ciruit mide 1 transitions to circuit mode 2, which then in turn comes back to circuit mode 1 in the next period of 
operation. There is only one energy storing element inductor L, therefore, the inductor current is the only state variable to watch for.

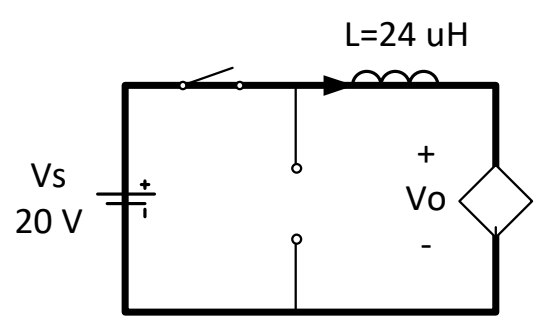

Mode 1

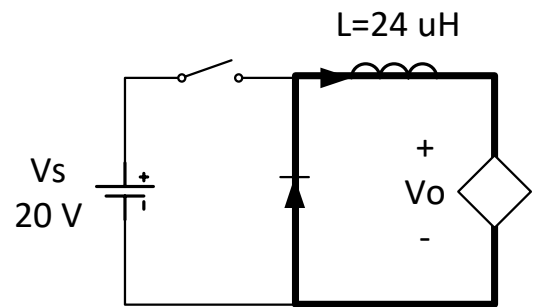

Mode 2

Fig. 6 Current flow in circuit modes

The simplified circuits of these modes are given below:

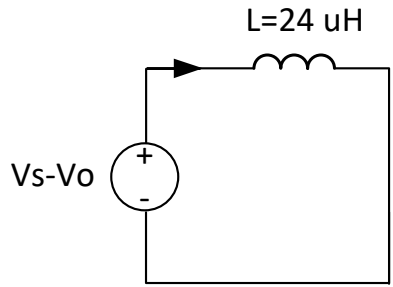

Mode 1

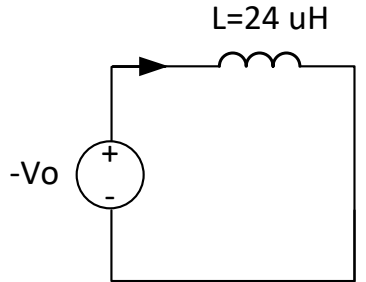

Mode 2

Fig. 7 Ssimplified circuits of the modes

The program declares inductor current $i_{L}$ as the state variable object, calls a function cktmode1 which uses the object in two circuit modes to determine current waveform over a switching period in steady state.

Circuit mode 1:

$$
\begin{aligned}
& \frac{d i_{L}}{d t}=+\frac{1}{L}\left(V_{S}-V_{O}\right) \\
& I_{L}=\frac{1}{s L}\left(V_{S}-V_{O}\right)+i_{L 0} \\
& \mathrm{~A} 1=0, \quad \mathrm{~B} 1=\frac{1}{L}\left(V_{S}-V_{O}\right), \quad \text { Ing } 1=i_{L 0}
\end{aligned}
$$

Circuit mode 2:

$$
\begin{aligned}
& \frac{d i_{L}}{d t}=+\frac{1}{L}\left(-V_{O}\right) \\
& I_{L}=\frac{1}{s L}\left(-V_{O}\right)+i_{L 1} \\
& \mathrm{~A} 2=0, \quad \mathrm{~B} 2=\frac{1}{L}\left(-V_{O}\right), \quad \text { Ing2 }=i_{L 1}
\end{aligned}
$$

Please note that this example has very simple circuit modes with the circuit matrix $A=0$, but the procedure enunciated, is the same for any converter with complicated circuit modes including multiple energy storage elements and non-zero circuit matrices.

\section{\%Part 2}

$\%$ Circuit mode 1 operation

$\mathrm{A} 1=0 ; \mathrm{B} 1=(1 / \mathrm{L}) *(\mathrm{Vs}-\mathrm{Vo})$;

Ing1= state (iL0, 0);

Eg1=state(nan, D*Ts);

iL1=cktmode1(Ing1, A1, B1, Eg1, dt);

is $1=\mathrm{iL} 1$; \%mode 1 configuration data

\%Ingress state at the beginning of circuit mode 1

\%to be determined Egress state at the end of circuit mode 1

\%calculate inductor current vectors in mode 1

\%calculate the source current vectors in mode 1 
\%Circuit mode 2 operation

A2=0; B2= $(1 / \mathrm{L}) *(-\mathrm{Vo})$; $\quad$ \% mode 2 configuration data

Ing2=state(iL1.mag(end), iL1.time(end)); \%Ingress state at the beginning of circuit mode 2

Eg2=state(nan, Ts); $\quad$ \%to be determined Egress state at the end of circuit mode 2

\%Note that Eg2.magnitude must be equal to the Ing1. magnitude of the circuit mode 1, after one period Ts

iL2=cktmode1(Ing2, A2, B2, Eg2, Ts); \%calculate inductor current vectors in mode 2

is $2=\mathrm{iL} 2$;

is2.mag=zeros(size(is2.mag));

\%calculate source current vectors in mode 2

iL.mag=[iL1.mag, iL2.mag];

\%the source current is zero in mode 2

iL.time=[iL1.time, iL2.time];

is.mag=[is1.mag, is2.mag];

\%inductor current in one full switching period

is.time $=[$ is1.time, is2.time];

plot(iL.time, iL.mag, 'linewidth', 2)

plot(is.time, is.mag, 'linewidth', 2)

\section{iL vs time}

is vs time

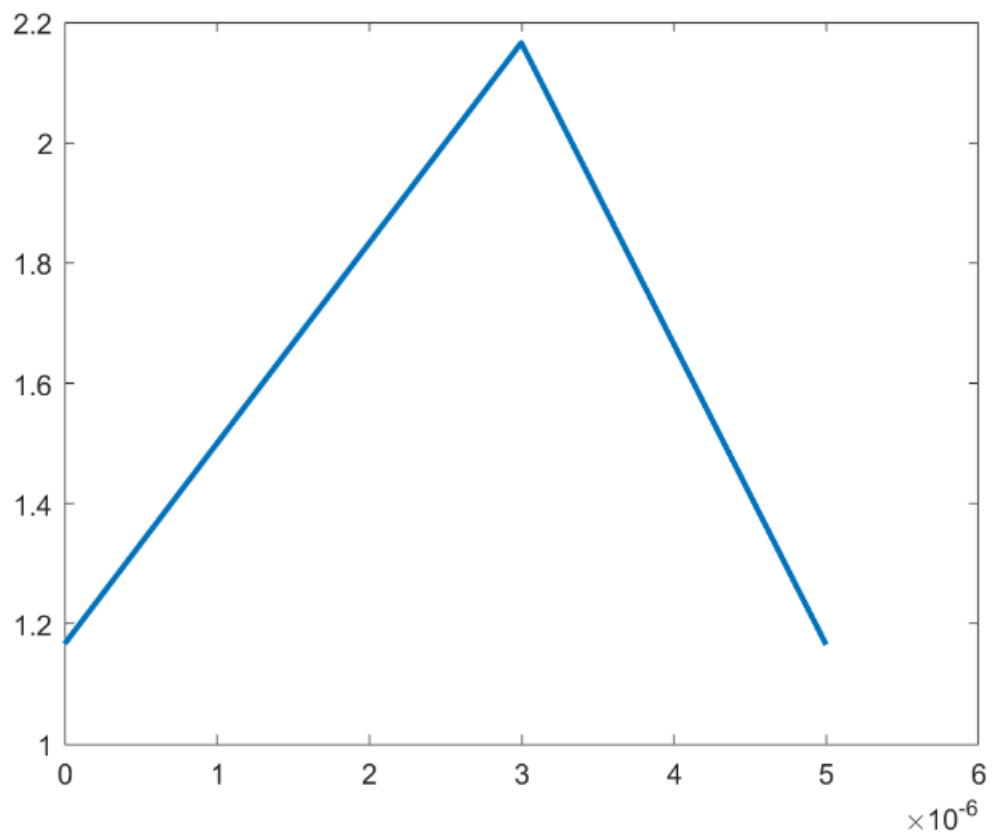




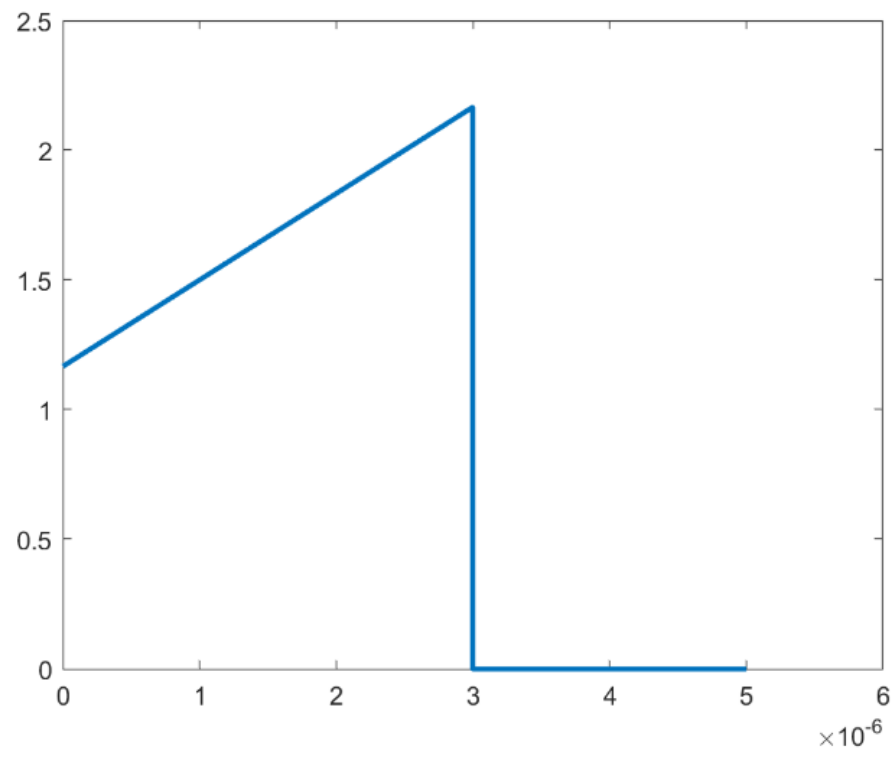

Fig. 8 a) Inductor current $i_{L}$

Fig. 8 b) Source current is

\subsubsection{Buck Converter in Discontinuous Conduction Mode (DCM) Operation (Output Power $P_{O}=5$ Watts)}

The Buck converter in DCM operates in three configurations: circuit mode 1 when the switch is turned on and remains on. The second circuit configuration in circuit mode 2 occurs when the switch is turned off for the duration of (1-D)Ts. The third circuit configuration in circuit mode 3 occurs when the inductor current becomes zero and remains zero during the off period of the switch. The circuit configuration and the current flow in circuit mode 1 is shown by the thick dark line in the first circuit in Fig. 6. The circuit configuration and the current flow in circuit mode 2 is shown by the thick dark line in the second circuit in Fig. 6. Ciruit mode 1 transitions to circuit mode 2 and then to circuit mode 3, which then in turn comes back to circuit mode 1 in the next period of operation. There is only one energy storing element inductor L, therefore, the inductor current is the only state variable to watch for.

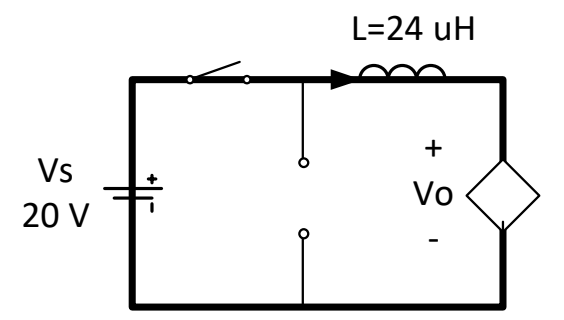

Mode 1

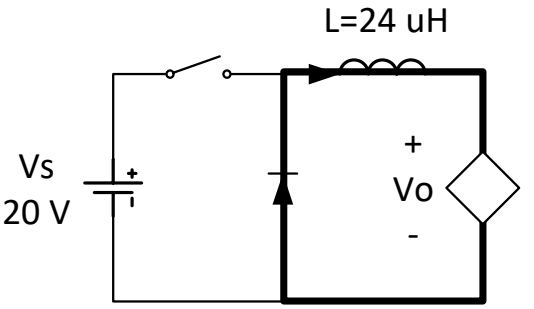

Mode 2

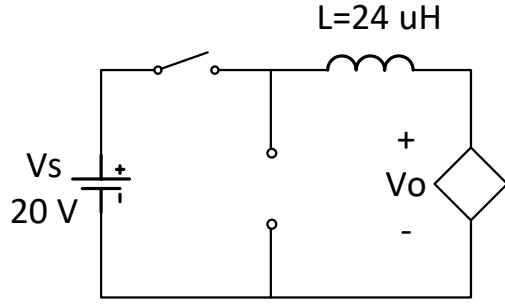

Mode 3

Fig. 9 Circuit modes in DCM operation of the Buck Converter

Modify the MATLAB code in part 2 by adding the following lines:

\%Part 2

$\cdot$

\%Circuit mode 3 operation

$\mathrm{A} 3=0 ; \mathrm{B} 3=0$; 
Ing3=state(iL2.mag(end), iL2.time(end));

Eg3=state(nan, Ts);

iL3=cktmode1(Ing3, A3, B3, Eg3, Ts);

is3=iL3;

iL.mag=[iL1.mag, iL2.mag, iL3.mag];

iL.time=[iL1.time, iL2.time, iL3.time];

is.mag=[is1.mag, is2.mag, is3.mag];

is.time=[is1.time, is2.time, is3.time];

plot(iL.time, iL.mag, 'linewidth', 2)

\%calculate inductor current vectors in mode 2

plot(is.time, is.mag, 'linewidth', 2)

\%inductor current in one full switching period

\%source current in one full switching period

\%the inductor current waveform in one full switching period

\%the source current waveform in one full switching period
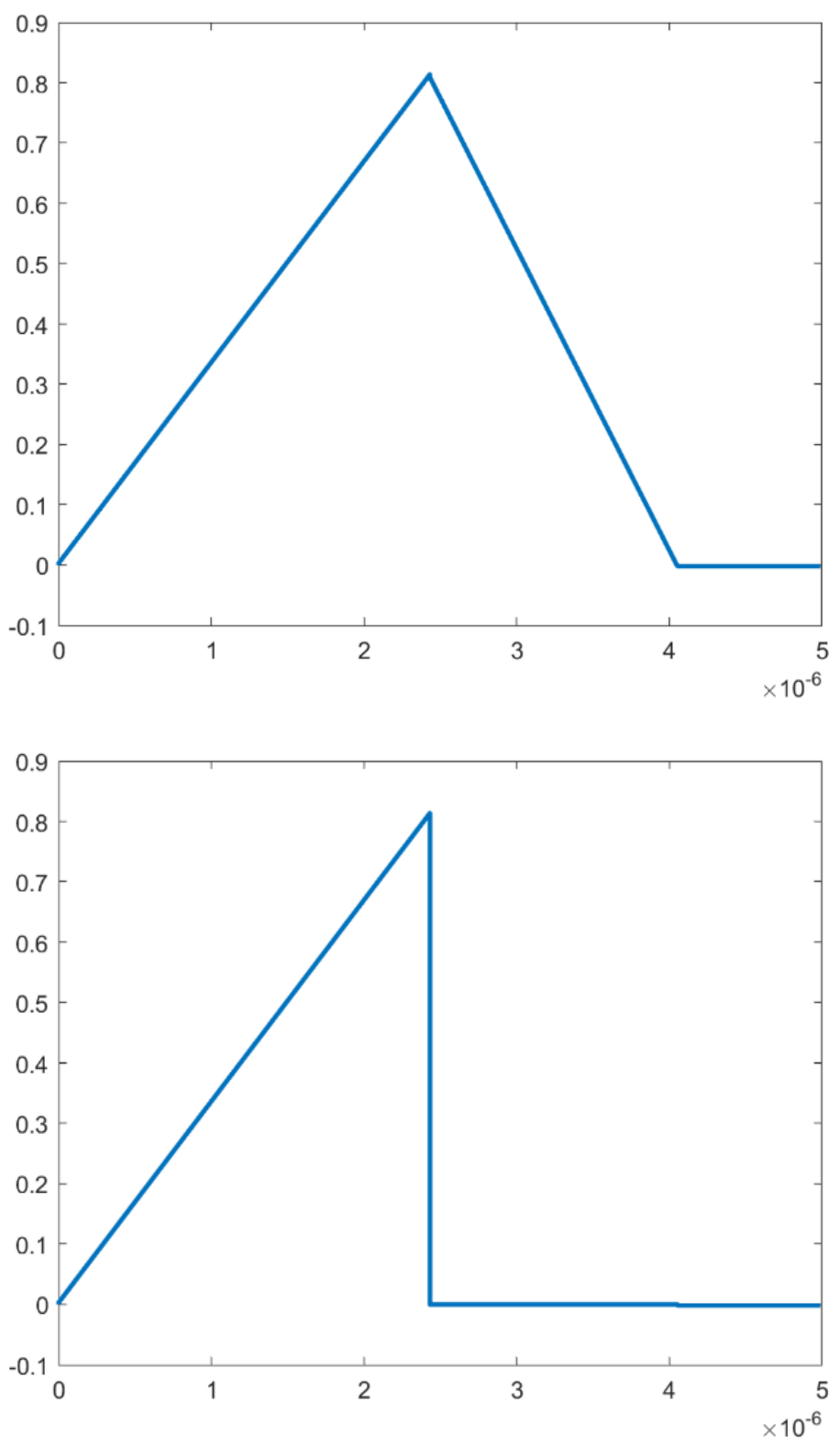

iL vs time

Fig. 10 a) Inductor current $i_{L}$ is vs time

Fig. 10 b) Source current is 


\subsection{Example of Flyback DC-DC Converter in CCM}

The flyback dc-dc converter is essentially a BuckBoost Converter with the inductor replaced by a transformer. The transformer provides an additional feature of electrical isolation between the input source and the output sink (load). The magnetizing inductance of the primary winding of the transformer serves as the energy storage element in the converter.

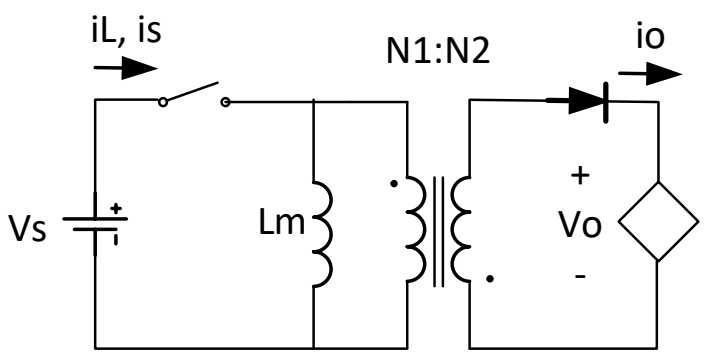

Fig. 11 Flyback Converter Topology

The following example shows a $24 \mathrm{~V}$ to 5V DC-DC Flyback Converter operating at $40 \mathrm{kHz}$ and a transformer with the primary inductance of $500 \mu \mathrm{H}$ and turn ratio of primary to secondary, $\mathrm{N}_{1} / \mathrm{N}_{2}$ of $3: 1$. Find the duty ratio $\mathrm{D}$ at which the converter must operate to deliver $5 \mathrm{~W}$ power to the resistive load at a voltage of $5 \mathrm{~V}$.

$\begin{array}{llll}\text { DC Output Current: } & I_{O d c}=\frac{P_{O}}{V_{O}} & \text { DC Source Current: } & I_{S d c}=\frac{P_{O}}{V_{S}} \\ \text { Load Resistance: } & R_{L}=\frac{V_{O}^{2}}{P_{O}} & \text { DC Inductor current: } & I_{L m d c}=\frac{I_{S d c}}{D}\end{array}$

To determine if the converter would operate in CCM or in DCM, we should calculate the load resistance at the

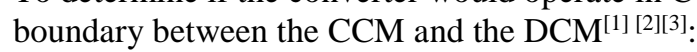

$$
R_{L B}=\frac{2 L m f_{S}}{(1-D)^{2}}
$$

Next, we determine the duty ratio and the initial inductor current in the circuit mode $1^{[1]}{ }^{[2]}$.

The converter operates in CCM if $R_{L} \leq R_{L B}$

Duty ratio for the desired output voltage is:

$$
D=\frac{V_{O}}{V_{O}+V_{S} \frac{N_{2}}{N_{1}}}
$$

Peak to peak ripple current in the inductor ${ }^{[1][2]}$ :

$$
I_{r p p}=\frac{V_{S} D}{\operatorname{Lm} f_{S}}
$$

Initial value of the inductor current in the circuit mode 1: $\quad I_{L m 0}=I_{L m d c}-\frac{I_{r p p}}{2}$ 
The converter operates in circuit mode 1 when the switch is closed and the magnetizing inductance Lm is energized by the voltage source Vs. The induced voltage on the secondary winding is such that it reverse biases the diode and therefore, no current in the voltage sink. In circuit mode 2 when the switch is opened and the magnetizing inductance Lm transfers energy to the transformer such that it forward biases the diode and therefore, current flows in the voltage sink.

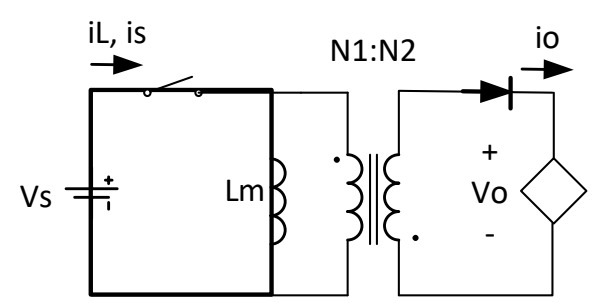

Mode 1

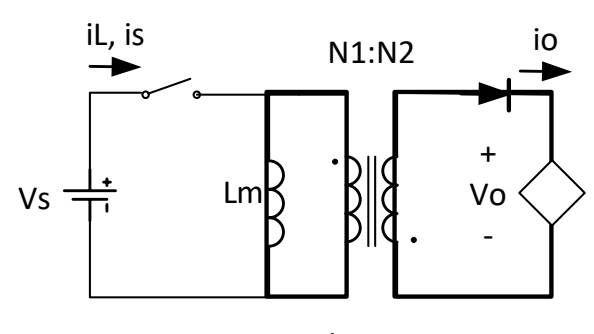

Mode 2

Fig. 12 Circuit modes in the steady state operation of the Flyback Converter

The simplified circuits of the modes are given below:

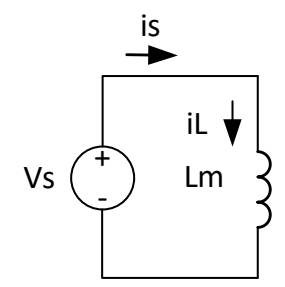

Mode 1

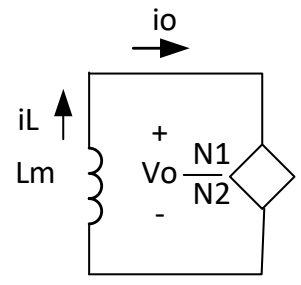

Mode 2

Fig. 13 Simplified circuit modes in the Flyback Converter

Circuit mode 1:

Circuit mode 2:

\%Flyback_CCM_2019_12_13.mlx

\%Part 1

Vs=24; Vo=5; Po=20; Lm=500e-6; fs=200e3; N1N2=3;

\%Part 2

\%Circuit mode 1 operation

$\mathrm{A} 1=0 ; \mathrm{B} 1=(1 / \mathrm{Lm}) * \mathrm{Vs}$;

Ing1=state(iLm0, 0);

$$
\begin{aligned}
& \frac{d i_{L m}}{d t}=+\frac{1}{L m}\left(-V_{O} \frac{N_{1}}{N_{2}}\right) \\
& I_{L m}=\frac{1}{S L m}\left(-V_{O} \frac{N_{1}}{N_{2}}\right)+i_{L 1} \\
& \mathrm{~A} 2=0, \quad \mathrm{~B} 2=\frac{1}{L m}\left(-V_{O} \frac{N_{1}}{N_{2}}\right), \quad \text { Ing2 }=i_{L m 1}
\end{aligned}
$$


Eg1=state(nan, tau);

iLm1=cktmode1(Ing1, A1, B1, Eg1, dt); is $1=\mathrm{iLm} 1$;

\%Circuit mode 2 operation

$\mathrm{A} 2=0 ; \mathrm{B} 2=(1 / \mathrm{Lm}) *\left(-\mathrm{Vo}^{*} \mathrm{~N} 1 \mathrm{~N} 2\right)$;

Ing2=state(iLm1.mag(end), iLm1.time(end));

Eg2=state(nan, Ts);

iLm2=cktmode1(Ing2, A, B2, Eg2, dt);

is $2=\mathrm{iL} 2$;

is2.mag=zeros(size(iLm2.mag));

iLm.mag=[iLm1.mag, iLm2.mag];

iLm.time $=[i L m 1$.time, iLm2.time $]$;

is.mag $=$ [is1.mag, is2.mag];

is.time $=$ [is1.time, is2.time];

plot(iLm.time, iLm.mag, 'linewidth', 2)

axis([0, Ts, 0, 2.4 ]); title('iL vs time')

plot(is.time, is.mag, 'linewidth', 2)

$\operatorname{axis}([0$, Ts, $0,2.4]) ; \quad$ title('is vs time') \%inductor current vector (object) in Mode 1

\%source current vector (object) in Mode 1

\%inductor current vector (object) in Mode 2

\%source current vector (object) in Mode 2

\%inductor current vector (object) in one full switching period

\%source current vector (object) in one full switching period

\%source current vector (object) in one full switching period

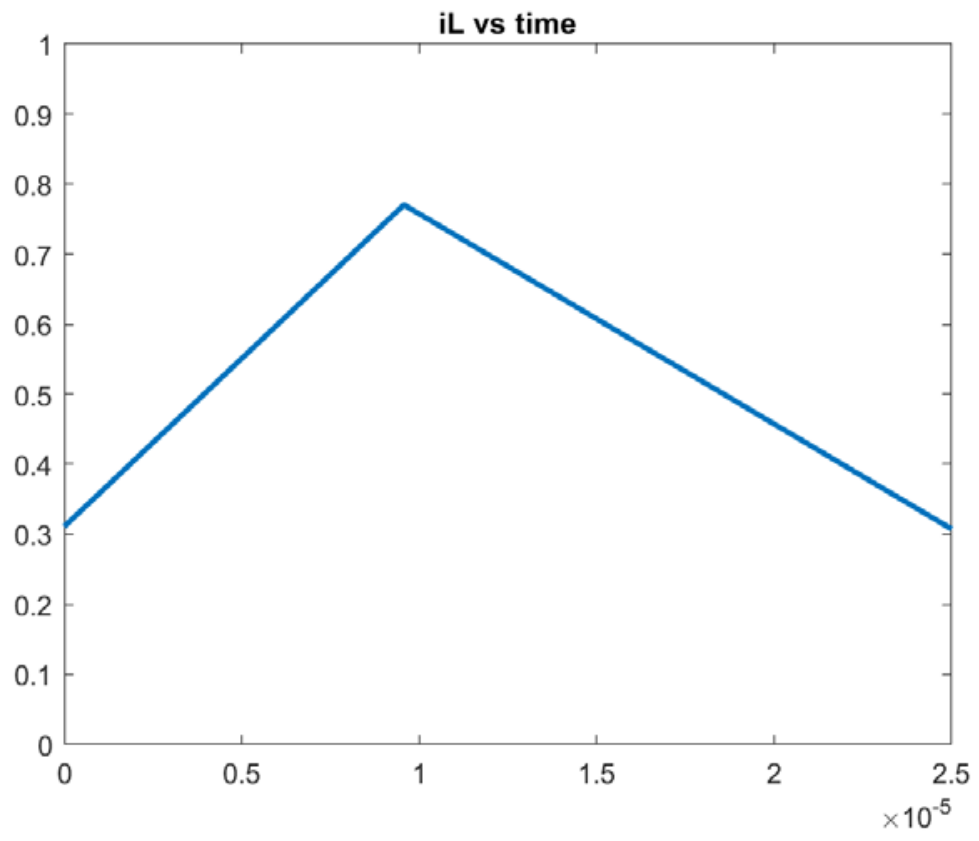




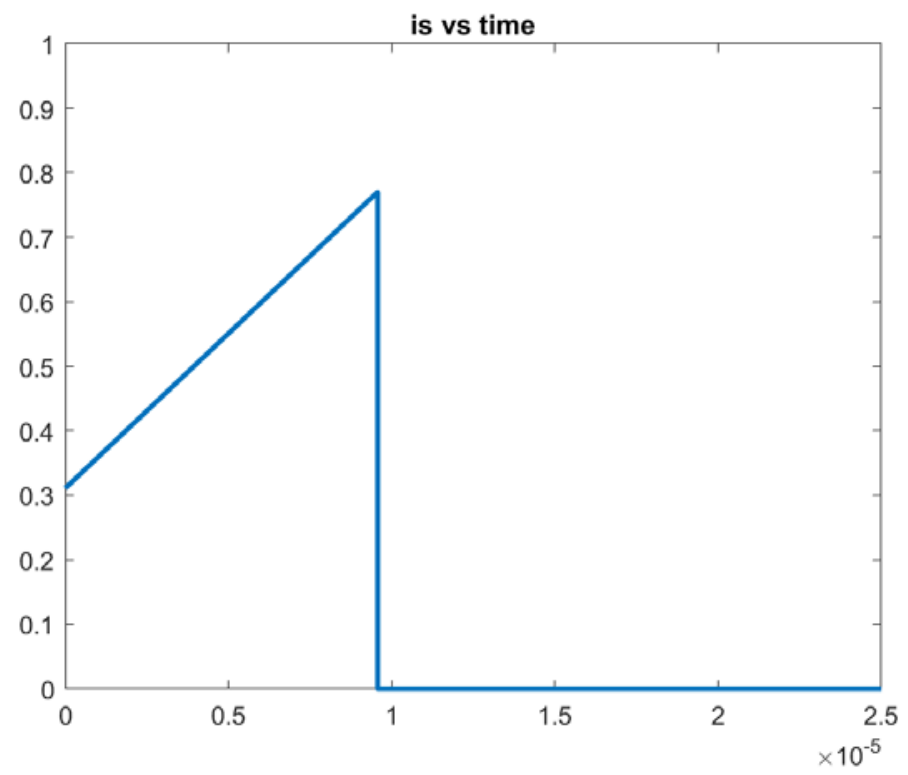

Fig. 14 a) Inductor current $i_{L}$

Fig. 14 b) Source current is

\section{CONCLUSION}

The paper presents the programming methodology for generating voltage and current waveforms in the steady-state analysis of Single Source Single Output (SISO) power converters. Use of state variable analysis and MATLAB increases understanding of the circuit operation. In this method, the power electronic converter operation is first resolved into circuit modes (states) based on the specifications and switching strategy employed. The inductor currents and capacitor voltages are used as objects with properties and methods in each circuit state.

Even though the Spice simulation is a well-known method of obtaining the time-domain waveforms of voltages and currents, it does not provide enough insight in the circuit operation. Since the power converters contain a network of switches which may assume multiple configurations giving rise to piece-wise configurable circuits and transitions among them. It is extremely important to identify the circuit configurations, the sequence and the modus operandi of transitions from one configurations to another. The overall voltage and current waveforms on all components including the switches are extremely useful in obtaining the average, peak ratings and stresses. This paper presents a methodical analysis of the circuit configurations and proposes a generalized MATLAB based method for generating the time domain waveforms in the steady state operation of converters. We employ state variable methods for analyzing the circuit operation.

\section{Reference}

[1] Ned. Mohan, Tore M. Undeland and William P. Robbins, "Power Electronics: Converters, Applications, and Design”, $2^{\text {nd }}$ ed., John Wiley \& Sons, 1995.

[2] Jai P Agrawal, “Power Electronic Systems: Theory and Design”, Prentice Hall, 2001.

[3] Daniel W. Hart, "Power Electronics”, McGraw Hill, 2010. 